\title{
Analysing low-voltage grids using a behaviour based load profile generator
}

\author{
N. Pflugradt ${ }^{1}$, J. Teuscher ${ }^{2}$, B. Platzer ${ }^{1}$ and W. Schufft ${ }^{2}$ \\ ${ }^{1}$ Chair of Technical Thermodynamics \\ Faculty of Mechanical Engineering \\ Chemnitz University of Technology \\ Straße der Nationen 62, 09107 Chemnitz (Germany) \\ Phone/Fax number:+0049 371531 38745/+0049 371531 838745, e-mail: noah.pflugradt@mb.tu-chemnitz.de \\ ${ }^{2}$ Chair of Power and High-Voltage Engineering \\ Faculty of Electrical Engineering and Information Technology \\ Chemnitz University of Technology \\ Straße der Nationen 62, 09107 Chemnitz (Germany) \\ Phone/Fax number:+0049 371531 37752/+0049 371531 837752, e-mail: jens.teuscher@etit.tu-chemnitz.de
}

\begin{abstract}
This paper contains a behaviour based load profile generator to generate realistic load profiles for every single household in a low voltage grid. The results are compared with measurements and it is shown that adding up all the generated load profiles results in approximately the same load and curve as measured on the real substation. Based on this, the paper shows that the common approach of scaling down standard load profiles to the individual houses yields very different results than using realistic load profiles.
\end{abstract}

\section{Key words}

Load Profiles, Low-voltage Grids, Power Flow Simulation

\section{Introduction}

In the last years the requirements of low-voltage grids changed based on renewable energies and new consumers. (f.e. electric vehicles). At the moment the power flow in low-voltage grids is mostly unknown. The challenge is the simulation of the consumption of electrical energy in the different households. This simulation is an important fact to develop management structures for future micro grids. Currently there is only the possibility of using synthetic profiles which are based on the standard load profiles. But these profiles do not show the realistic consumption of households. Real load profiles of households are fluctuating depending on the usage of different devices.

Obtaining load profiles for every single household in an area is prohibitively expensive though, if possible at all. Using the behaviour based load profile generator is a quick and easy way of generating various realistic load profiles of different households thus creating a realistic simulation of the power flow in a low-voltage grid. This paper shows the feasibility of this approach and the differences between it and simulations with standard load profiles.

\section{Technical Background}

\section{A. Load Profiles for households}

For different uses exist different types of household load profiles. Based on their approach these profiles are divided in bottom-up-models and top-down-models. [1]

Important examples of the top-down-model are the standard load profiles. These are developed for modelling the energy consumption of a large number of households at the same time. They are primarily used by the Distribution System Operators. The System Operators use these profiles to buy energy.

Another use case is the simulation of power flow in lowvoltage grids. These simulations need either real, measured load profiles or very good approximations of such profiles. For this task the bottom-up-models are used. Mostly they are based on statistical or probabilistic processes, on measurements and on the behaviour of the consumer or the weather conditions. The bottom-upmethodologies use among other parameters the individual energy consumption of selected households and the technical properties of that household. Because individual load profiles are caused by the behaviour of real consumers, generating them requires taking into account all the constraints of both the behaviour of the consumers as well as the constraints of the devices.

Figure 1 shows one example for the differences between the synthetic standard load profiles and the real consumption of a household. 


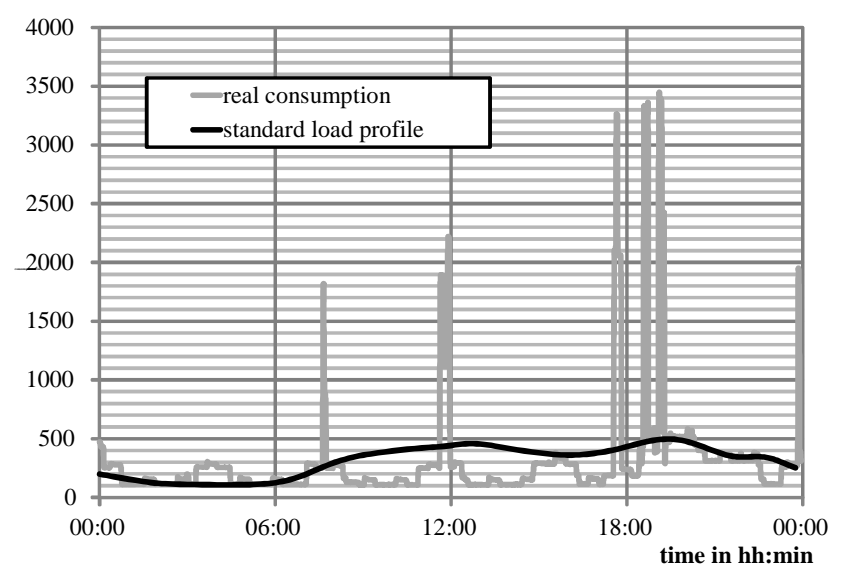

Fig. 1. Comparison between standard load profile and a real load profile of a household

\section{B. Simulation of low-voltage grids}

The simulated low-voltage grid $\left(\mathrm{U}_{\mathrm{r}}=0.4 \mathrm{kV}\right)$ in this paper is based on a real existing grid in Germany. [2] This grid is an urban grid which supplies a lot of households in a small area. In this paper the load profile generator is tested on a model of an urban low-voltage grid including almost 80 connection points over an area of $0.25 \mathrm{~km}^{2}$. An average connection point supplies approximately 10 households.

The program for the power flow simulation is done with the tool MATPOWER. [3] This is a static calculation program based on MATLAB. It simulates only one moment of the power flow. To calculate the power flow in the grid at various points in time with different loads, an extension for MATPOWER was developed.

Figure 2 shows another extension developed by the authors to interpret the simulated results. With this extension it is possible to find critical situations, f.e. a high utilization of the individual lines or the voltage drop in the grid at each time step.

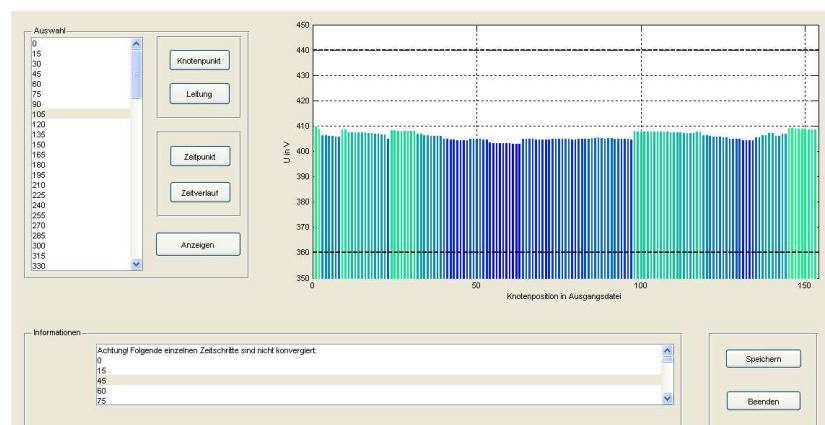

Fig. 2. Overview about the developed extension to interpret the simulated results

\section{Load Profile Generator}

For this analysis a new behaviour based load profile generator was used [4]. Most load profile generators according to [1] are based on statistical approaches or Markov chains.
One example is the program DHWCalc [5], a tool for generating load profiles for domestic hot water use. It works by assigning probabilities to time spans, for example ,50\% between 12:00 and 13:00 on Weekdays“. These probabilities are then used to randomly distribute hot water usage events over all timespans, based on a rather complicated algorithm. The algorithm takes into account differences in winter and summer usage behaviour, vacations and desired total yearly use.

Other load profile generators for electrical energy use more complicated algorithms. Examples include finite state machines or Markov chains, often coupled with probabilities. A very simplified example of this might be „After eating dinner there is an $80 \%$ chance of having the TV turned on between 18:00 and 19:00, combined with a $30 \%$ of turning on the DVD player". Those approaches yield good results for creating a load profile for many hundreds or thousands of households. But it is very difficult to correctly simulate a realistic load profile for a single household, especially for special cases like shift workers or families with children.

Consider for example calculating the amortization time of a battery used to raise the percentage of photovoltaic power used in-house. It makes a large difference if the household members are home during the day when the sun is shining, if they work at nights or if they take 3 weeks of vacation in the summertime just when there is the most sun.

The behaviour based load profile works differently: It uses a psychological model based on the works of D. Dörner [6] to simulate the behaviour of each member of the household. From this the load profile generator determines the device usage and then calculates the energy profile.

Dörner's work is based on the theory of the selfish homo oeconomicus. The basic assumption here is that every person always choses the action which best satisfies his or her desires.

Dörner came up with a way to represent this very basic idea with mathematical model in the computer: $\mathrm{He}$ modelled each desire as "leaking water tank". One of his graphics is shown in Fig. 3. This means that the desire to do something increases over time. It frequently needs to be satisfied. And there is a logarithmic increase in the importance of the desire the more urgent (empty) the desire gets. 


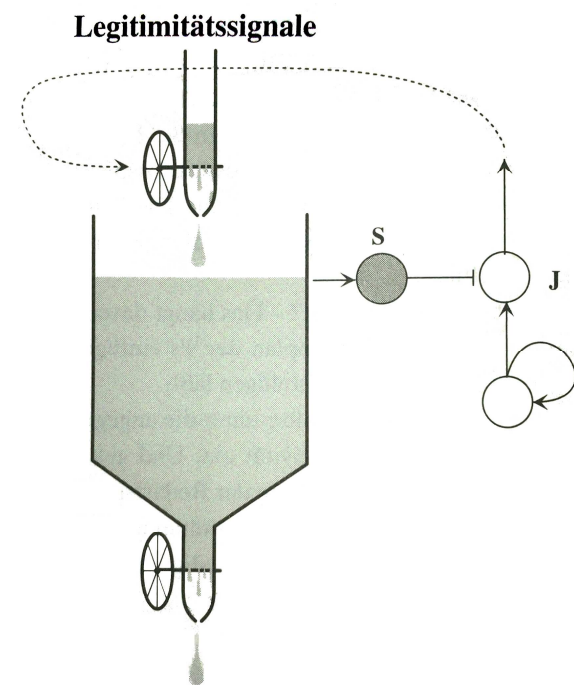

Fig. 3. The mental model Dörner uses for desires [6]

Dörner was able to fully explain human behaviour with only five desires. For example he says that going to work satisfies the desire to live (one needs money to buy food to survive), the desire to feel competent (that you can do your work shows you that you are competent at your work) and the desire to feel some amount of control over your surroundings, but not too much (you control your environment at work).

In real life, it is rather obvious that one has to go to work every day to earn money to buy food or to have some disposable income to indulge oneself. Putting that kind of planning into a simulation proved to be unfeasible though. Therefore a more simple desire model was used in the load profile generator.

Here the desires have been split apart into one desire for each activity group. An activity group is defined as activities that can replace each other. For example if the simulated person is hungry, she can eat bread, cook spaghetti or order pizza. Every activity will fill the desire to stop feeling hungry.

To fulfil the desires activities have to be available for the simulated person to choose from. For this a simulated household is constructed with various devices.

Based on the devices possible actions are available to the simulated person. For example having an oven makes it possible to bake a cake, make pizza or to cook a turkey. Of course each of these activities has a different duration and uses different amounts of energy.

Activities have a m:n relationship to devices. For example watching a DVD movie requires both the DVD player and the TV. Reading a book requires no devices at all.

For each device in each activity there is a load profile, based on measured data. One example is the device load profile shown in Fig 4.

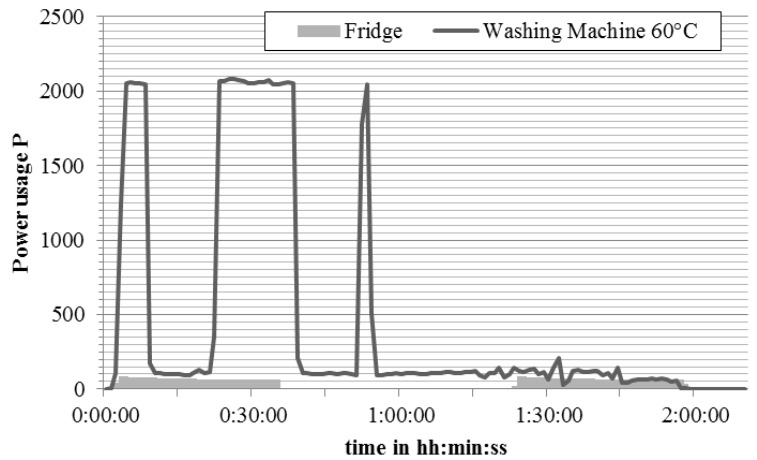

Fig. 4. Examples of the measured load profiles: A washing machine and a fridge.

The advantage of this rather complicated way of describing a household is that it's very flexible. In the load profile generator it's possible to simulate the energy use of a single student just as well as the energy use of a multi generation family living together in a big house.

And because the load profile generator provides a very flexible framework for modelling the behaviour, it's easy to make changes.

For example one might want to analyse what happens to the energy use when instead of working 8 hours per day the person switches to a part time job. In a load profile generator based on stochastic data this would mean recalculating the probabilities for all the devices. With the behaviour based load profile generator one just switches the duration of the job from 8 to 4 hours and the software calculates all the rest. Because the person is coming home earlier, he will probably cook at home and then based on the programmed desires spend the afternoon for example with watching TV.

Obviously there are huge variations in the behaviour of different people in different households. It was decided that instead of trying to model an "average" household, whatever that might be, it would be more useful to model various concrete examples. The examples were based on either personal experience or small surveys. The load profile generator contains more than 100 devices, more than 20 different households, is completely customizable and available for free under [7].

\section{Applications of the Load Profile Generator}

The purpose of the load profile generator is split in two major areas: One is providing load curves for simulating and testing hybrid systems and the other one is providing realistic load curves for a better understanding of the power flow in the grid.

Hybrid systems are systems that contain at least some sort of power generation unit and some sort of storage device. One example might be a photovoltaic array combined with a battery, a combination of battery, fuel cell and electrolyser or a fuel cell with a microcombined-heat-power-plant (micro-CHP-plant).

These combinations of various devices all have in common that their usefulness depends very much on the 
energy use in the household: If all power generated by the PV is used right away, the battery will be pointless.

On the other hand, if there is no power use during the day, then without the battery it won't be possible to keep the generated power from the day to the evening. So to properly size these devices and to ensure that the control algorithm are able to deal with the various problems like vacations, sudden illness with the associated higher energy use or even shift workers, realistic load profiles for a lot of different situations are needed.

The other application of the load profile generator is shown in this paper. For the future it is planned to use this approach to help with predicting the energy use in a household. This could work by automatically identifying the devices in a measured load profile [8] and estimating device usage. Furthermore, there are automatically finding parameters to generate a new household. This feature is not implemented yet though.

\section{Analysing a low-voltage grid}

Validating the load profiles is no easy task since there simply aren't any detailed, individual measurements available for a large number of households. The only measurements available to the authors for the task are from the measurement of a substation in 2010.

The task of determining the individual load profiles from that is a bit like determining if the number 10 was created by adding $5+5$ or $8+2$.

Due to the impossibility of this the following approach was used: First in cooperation with the local utility it was determined how much electrical energy was used in each house per year and how many households are in each house. Due to privacy concerns house numbers were randomized to protect the consumer. Because the intention of this paper is demonstrating the feasibility of the described approach the randomization does not matter in this case. To analyse a real low-voltage grid the real energy consumption would have to be used.

Based on the provided house data, the correct number of household profiles was generated with approximately the same amount of yearly energy use. After that the profiles for each household were added up to combined load profiles for each connection point.

The result of a) adding up all the load profiles and b) the measurement at the substation is shown in figure 5 .

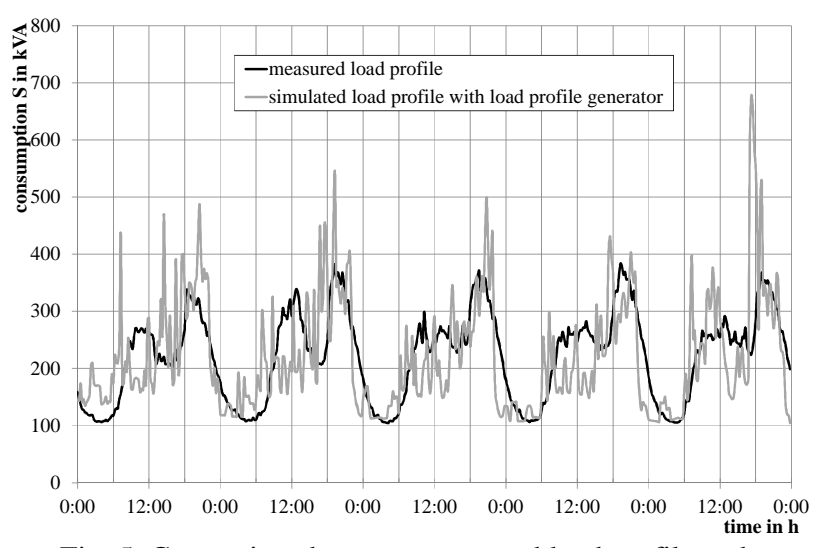

Fig. 5. Comparison between measured load profile and simulated profile on the substation

The interesting part is now the comparison of the power flow simulation when using the same, scaled down standard load profile at each house versus using the simulated load profiles. Figure 6 shows this comparison for households with the same energy consumption.

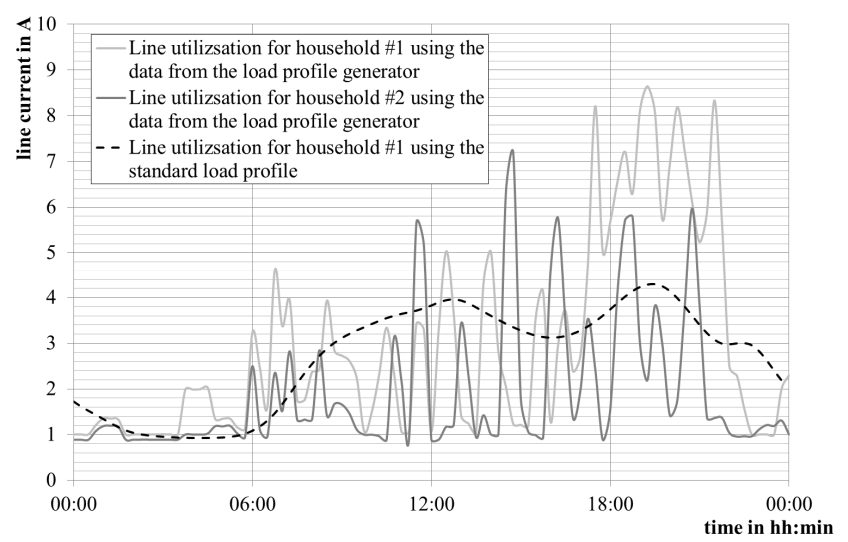

Fig. 6. Comparison between simulation of load profiles and the standard load profiles

Due to the varying load, previously assumed power reserves in the grid for charging electric vehicles might be a lot smaller than originally calculated and a charging management system will probably be needed a lot earlier.

In former works the capacity to charge electric vehicles depends on the utilization of the transformer [1]. With the simulated load profiles the utilization of the lines turns out to be an important issue too. Figure 3 shows this different line utilization. The simulated load profiles reduce the capacity for charging electric vehicles sometimes by half. This problem will be analysed in more detail in future research.

The situation becomes even more interesting when a large photovoltaic system is placed in one of the houses: While a simulation using the standard load profiles shows the house is dependable energy exporter on sunny days, the simulation with the more realistic simulated load profiles shows that it's rapidly oscillating between drawing power from the grid and providing power. While it's widely known that this happens for a single house, the result of what happens to the power flows in a grid in such cases is very important for substation planning. 


\section{Conclusion}

In this paper a new type of load profile generator was introduced. It has been used to generate individual load profiles for every single household attached to a substation.

It has been shown that adding up the simulated load profiles from the load profile generator yields a new load profile that is very close to measurement of the load profile on a substation. Therefore the individual load profiles and the method of adding up the profiles seems to be valid.

Using the individual load profiles for each household it is easily possible to do a realistic analysis of the power flows in a low voltage grid which yields considerably better results than simply assigning a scaled down standard load profile to each node in the grid.

Additionally it has been shown that using the scaled down standard load profiles in a grid with for example medium to large photovoltaic systems yields very different load flow results than using the standard load profiles.

\section{Acknowledgement}

This work is one part of the research group smart distributed power-storage systems and is supported by the European Social Fund.

\section{References}

[1] A. Grandjean, J. Adnot, G. Binet, A review and an analysis of the residential electric load curve models, Renewable and Sustainable Energy Reviews, Volume 16, Issue 9, December 2012, Pages 6539-6565, ISSN 1364-0321, 10.1016/j.rser.2012.08.013.

[2] J. Teuscher, A. Götz, W. Schufft: "Electric vehicles and their effects in low-voltage grids“, ICREPQ' 11. Las Palmas de Gran Canaria, 2011

[3] R.D. Zimmermann, C.E. Murillo-Sánchez: MATPOWER 4.1 User's Manual, Power Systems Engineering Research Center (PSERC), 2011

[4] Pflugradt, N.; Platzer, B.: Behavior based load profile generator for domestic hot water and electricity use: Innostock, 12th International Conference on Energy Storage, Lleida (Spain), 2012

[5] Jordan, U.; Vajen, K.: DHWcalc: Program to generate domestic hot water profiles with statistical means for user definied conditions. In: Goswami, D. Y. (Hrsg.): Proceedings of the 2005 Solar World Congress. Orlando, Florida, USA, August 6 - 12, 2005. Boulder, Colorado 2005.

[6] Dörner, D.: „Bauplan für eine Seele“, RowohltTaschenbuch-Verl, Reinbek bei Hamburg, 2008, ISBN 3499611937

[7] Pflugradt, N: Online-Load-Profil-Generator, www.tuchemnitz.de/ noah, 22.10.2012

[8] Hart, G. W.: Nonintrusive appliance load monitoring, In: Proceedings of the IEEE 80 (12), S. 1870-1891., 1992 